\title{
The association between microbial exposure and indoor climate in Danish nursing homes
}

\author{
Pil Uthaug Rasmussen ${ }^{\text {a* }}$, Katrine Uhrbrand ${ }^{\mathrm{a}}$, Anne Mette Madsen ${ }^{\mathrm{a}}$ \\ National Research Centre for the Working Environment, Lersø Parkallé 105, 2100 Copenhagen Ø, Denmark
}

\begin{abstract}
Humans are exposed to microorganism in most environments, including indoors. In this study, we examined the occupational exposure to bacteria and fungi and its correlation with indoor temperature and relative humidity for people employed in five nursing homes in the Capital Region of Denmark. We found negative correlations between temperature and fungal and bacterial exposures in personal air, sedimented dust, and surface samples, whereas we found positive correlations between bacteria and relative humidity for indoor and outdoor air samples. Our findings seem to match that of other studies, which have found strong correlations between temperature and relative humidity on the concentrations of microorganisms.
\end{abstract}

Peer-review under the responsibility of the organizing committee of the ICMB21.

Keywords: bacteria; bioaerosols; fungi; healthcare worker; mould; occupational health; Staphylococcus

\section{Introduction}

Microorganism are present in all environments, including indoor environments. However, in some environments, exposures to microorganisms and the presence of pathogenic and problematic microorganisms may be elevated. High concentrations of microorganisms and certain problematic bacteria and fungi can lead to health related problems, such as infections, inflammation of the airways, asthma, and allergies. The risk associated with exposure to microorganisms is especially a problem for immunocompromised individuals such as the elderly. Nursing homes may thus be a higher source of exposure to microorganisms for the staff members than typical indoor or office environments. However, the survival of microorganisms is closely linked to temperature and relative humidity, but whether microbial exposures in nursing homes are affected by the indoor climate, comparable to typical homes, remains to be seen. The aim of the study was therefore to investigate whether the occupational exposure to microorganisms in nursing homes might correlate to the indoor climatic environment.

\section{Methodology}

To investigate the occupational exposure of nursing home staff, five nursing homes were visited in the Capital Region of Denmark. Sampling was done on two days (14 days apart) and on each day a range of samples were taken: personal air samples were taken using GSP (Gesamtstaubprobenahme) samplers, stationary air samples were taken using GSP and six-stage ACI (Andersen Cascade Impactor) samplers, sedimented dust samples were taken using EDCs (electrostatic dust collectors), and surface samples were taken with moistened swabs (eSwabs). Sampling was done in both common rooms and in rooms with residents known to be colonized with methicillin-resistant Staphylococcus aureus (MRSA), as we in a previous project examined the occupational exposure to MRSA in the same samples [1]. Outdoor reference samples were taken using GSP samplers. Temperature and relative humidity was measured using data loggers (Tinytag Plus).

ACI samples were sampled directly on nutrient agar and later incubated at $25^{\circ} \mathrm{C}$ for quantification of the bacterial exposure and/or on SaSelect agar and incubated at $37^{\circ} \mathrm{C}$ for quantification of staphylococci, while the remaining samples were extracted and grown in serial dilutions on nutrient agar $\left(25^{\circ} \mathrm{C}\right)$ and SaSelect agar $\left(37^{\circ} \mathrm{C}\right)$. Personal exposure samples were further plated on dichloran glycerol agar (DG-18) and incubated at $37^{\circ} \mathrm{C}$ for quantification of pathogenic fungi. Bacteria and fungi were identified using MALDI-TOF MS, and bacteria grown on SaSelect agar were further divided into staphylococci and non-staphylococci bacteria. From personal air samples, endotoxin levels were determined using the kinetic limulus amoebocyte lysate test. From the six-stage ACI the aerodynamic diameter was calculated. Correlations were determined using Pearson's product moment correlation on both the concentrations and log transformed concentrations.

\footnotetext{
* Corresponding author. +45 39165200 pur@nfa.dk
} 


\section{Results}

\subsection{Microbial community and exposure}

Around 100 different bacterial species from 27 different genera were identified across the different sample and agar types. In personal exposure samples, the genera most often found were Micrococcus, Staphylococcus and Corynebacterium. For fungi, 13 species were identified from five different genera, the most common being Aspergillus, especially A. niger and A. fumigatus. The general exposure to endotoxin seemed to vary greatly between staff members (geometric mean 1.94 EU/m3; ranging from $0.02-$ $\left.59.00 \mathrm{EU} / \mathrm{m}^{3}\right)$.

\subsection{Temperature}

Negative correlations between temperature and fungi incubated at $37^{\circ} \mathrm{C}$ were found in the personal exposure measurements $(\mathrm{r}$ $=-0.38, p=0.03$ ). For bacteria, negative correlations between temperature and bacterial concentrations were found in EDC samples, for bacteria grown on nutrient agar at $25^{\circ} \mathrm{C}(\mathrm{r}=-0.32, \mathrm{p}=0.03$, log transformed: $\mathrm{r}=-0.29, \mathrm{p}=0.05)$, for all bacteria grown on SaSelect agar at $37^{\circ} \mathrm{C}(\mathrm{r}=-0.34, \mathrm{p}=0.02$, log transformed: $\mathrm{r}=-0.40, \mathrm{p}=0.01)$, and for only the staphylococci grown on SaSelect agar $(r=-0.40, p=0.05)$. In addition, a negative correlation between temperature and the non-staphylococci grown on SaSelect agar was found in surface samples (log transformed: $r=-0.34, p=0.01$ ).

\subsection{Relative humidity}

A positive correlation was found between non-staphylococci grown on SaSelect agar at $37^{\circ} \mathrm{C}$ and relative humidity for the sixstage ACI samples $(r=0.36, p=0.04$; log transformed: $r=0.34, p=0.05)$. Similarly, we found a strong positive correlation between the non-Staphylococci grown on SaSelect agar and relative humidity in outdoor samples $(r=0.83, p=0.04 ; \log$ transformed: $\mathrm{r}=0.88, \mathrm{p}=0.02$ ).

The aerodynamic diameter and endotoxin concentration were not correlated to neither temperature nor relative humidity.

\section{Discussion}

We found negative correlations between temperature and the concentration of fungi and bacteria - for bacteria this both included staphylococci and non-staphylococci. We further found a positive correlation between relative humidity and bacterial concentrations, both in indoor and outdoor samples. These findings are similar to that of Zhu et al. [2], who studied the indoor and outdoor bacterial concentrations in office buildings equipped with air conditioning in Arizona, USA, and related that to measured environmental variables. They found that relative humidity had the most pronounced influence on bacterial concentrations both indoor and outdoor, where higher relative humidity lead to higher bacterial concentrations. Furthermore, they found, similar to here, a negative correlation between temperature and bacterial concentrations. Madsen et al. [3] similarly found an impact of temperature in indoor air on Staphylococcus concentrations, and that the effect of environmental variables such as temperature differed among bacterial genera. Results matching this has also been found for fungi in a study by Ren et al. [4]. Here the authors found that the impact of temperature and relative humidity in indoor air differed among fungal genera. Future studies may therefore examine whether impacts of temperature and relative humidity differ among problematic bacterial and fungal species and genera. However, such analyses may depend on whether the taxa studied can be found in enough samples in order to make sound correlations. If such patterns are found, it may be a potential way to reduce occupational as well as general indoor exposure to certain problematic bacteria and fungi.

\section{References}

[1] Rasmussen, P., Uhrbrand, K., Bartels, M. D., Neustrup, H., Karottki, D. G., Bültmann, U., Madsen, A. M. (2021). Occupational risk of exposure to MRSA and the quality of infection hygiene in nursing homes. Frontiers of Environmental Science \& Engineering 15(3): 41.

[2] Zhu, H., P. E. Phelan, T. Duan, G. B. Raupp, H. J. S. Fernando, Che, F. (2003). Experimental study of indoor and outdoor airborne bacterial concentrations in Tempe, Arizona, USA. Aerobiologia 19(3): 201-211.

[3] Madsen, A. M., S. Moslehi-Jenabian, M. Z. Islam, M. Frankel, M. Spilak, Frederiksen, M. W. (2018). Concentrations of Staphylococcus species in indoor air as associated with other bacteria, season, relative humidity, air change rate, and S. aureus-positive occupants. Environmental Research 160: $282-291$.

[4] Ren, P., T. M. Jankun, K. Belanger, M. B. Bracken, Leaderer, B. P (2001). The relation between fungal propagules in indoor air and home characteristics. Allergy 56(5): 419-424. 